\title{
An additive problem with primes and almost-primes
}

by

\author{
T. P. Peneva and D. I. Tolev (Plovdiv)
}

1. Introduction. In 1937 I. M. Vinogradov [10] proved that for every sufficiently large odd integer $N$ the equation

$$
p_{1}+p_{2}+p_{3}=N
$$

has a solution in prime numbers $p_{1}, p_{2}, p_{3}$.

Two years later van der Corput [9] used the method of Vinogradov and established that there exist infinitely many arithmetic progressions consisting of three different primes. A corresponding result for progressions of four or more primes has not been proved so far. In 1981, however, D. R. HeathBrown [5] proved that there exist infinitely many arithmetic progressions of four different terms, three of which are primes and the fourth is $\mathrm{P}_{2}$ (as usual, $\mathrm{P}_{r}$ denotes an integer with no more than $r$ prime factors, counted according to multiplicity). One of the main points in [5] is a result of BombieriVinogradov's type for the sum

$$
\sum_{\substack{x<p_{2}, p_{3} \leq 2 x \\ p_{1}+p_{3}=2 p_{2} \\ p_{2}-2 p_{3} \equiv 0(\bmod d)}} u\left(p_{1}\right) u\left(p_{2}\right) u\left(p_{3}\right),
$$

where $d$ is squarefree, $(d, 6)=1 ; u(n)=(\log n) / \log 3 x$ for $n \geq 5$ and $u(n)=0$ otherwise.

Recently Tolev [8] found an analogous result for the quantity

$$
J_{k, l}(N)=\sum_{\substack{p_{1}+p_{2}+p_{3}=N \\ p_{1} \equiv l(\bmod k)}} \log p_{1} \log p_{2} \log p_{3},
$$

where $N$ is a sufficiently large odd integer and $(l, k)=1$. In [8] the HardyLittlewood circle method and the Bombieri-Vinogradov theorem were applied, as well as some arguments belonging to H. Mikawa.

1991 Mathematics Subject Classification: Primary 11N36. 
It would be interesting to prove that there exist infinitely many arithmetic progressions of three different primes such that for two of them, $p_{1}$ and $p_{2}$, say, both the numbers $p_{1}+2, p_{2}+2$ are almost-primes. In the present paper we study this problem. Our main tool is a result of BombieriVinogradov's type which we establish using the method developed in [8].

Let $x$ be a sufficiently large real number and $k_{1}, k_{2}$ be odd integers. Denote by $D_{k_{1}, k_{2}}(x)$ the number of solutions of the equation

$$
p_{1}+p_{2}=2 p_{3}
$$

in primes $p_{1}, p_{2}, p_{3}$ such that

$$
x<p_{1}, p_{2}, p_{3} \leq 3 x
$$

and

$$
p_{1}+2 \equiv 0\left(\bmod k_{1}\right), \quad p_{2}+2 \equiv 0\left(\bmod k_{2}\right) .
$$

Let us also define

$$
\gamma(x)=\sum_{\substack{x<m_{1}, m_{2}, m_{3} \leq 3 x \\ m_{1}+m_{2}=2 m_{3}}} \frac{1}{\log m_{1} \log m_{2} \log m_{3}}, \quad \sigma_{0}=2 \prod_{p>2}\left(1-\frac{1}{(p-1)^{2}}\right) .
$$

We prove the following

Theorem. For each $A>0$ there exists $B=B(A)>0$ such that

$$
\sum_{\substack{k_{1}, k_{2} \leq \sqrt{x} /(\log x)^{B} \\\left(k_{1} k_{2}, 2\right)=1}}\left|D_{k_{1}, k_{2}}(x)-\frac{\sigma_{0} \gamma(x)}{\varphi\left(k_{1}\right) \varphi\left(k_{2}\right)} \prod_{p \mid\left(k_{1}, k_{2}\right)} \frac{p-1}{p-2}\right| \ll \frac{x^{2}}{(\log x)^{A}} .
$$

For squarefree odd $k$ we define $J_{k}(x)$ as the number of solutions of the equation (1) in primes satisfying (2) and such that

$$
\left(p_{1}+2\right)\left(p_{2}+2\right) \equiv 0(\bmod k) .
$$

The Theorem stated above implies

Corollary 1. For each $A>0$ there exists $B=B(A)>0$ such that

$$
\sum_{\substack{k \leq \sqrt{x} /(\log x)^{B} \\(k, 2)=1}} \mu^{2}(k)\left|J_{k}(x)-\sigma_{0} \gamma(x) \prod_{p \mid k} \frac{2 p-5}{(p-1)(p-2)}\right| \ll \frac{x^{2}}{(\log x)^{A}} .
$$

REMARK. We shall prove the Theorem and Corollary 1 with $B=$ $16 A+100$.

Using Corollary 1 we get 
COROLlary 2. There exist infinitely many triples $p_{1}, p_{2}, p_{3}$ of distinct primes such that $p_{1}+p_{2}=2 p_{3}$ and $\left(p_{1}+2\right)\left(p_{2}+2\right)=\mathrm{P}_{9}$.

2. Notations. Let $x$ be a sufficiently large real number and $A$ a positive constant. The constants in $\mathcal{O}$-terms and $\ll$-symbols are absolute or depend only on $A$. We shall denote by $m, n, d, d_{1}, d_{2}, a, q, k, k_{1}, k_{2}, l, r, h, f$ integers, by $p, p_{1}, p_{2}, p_{3}$ prime numbers and by $y, z, t, \alpha$ real numbers. As usual $\mu(n), \varphi(n)$ denote Möbius's function and Euler's function; $\tau_{k}(n)$ is the number of integer solutions of the equation $d_{1} \ldots d_{k}=n ; \tau(n)=\tau_{2}(n)$. We denote by $(m, n)$ and $[m, n]$ the greatest common divisor and the least common multiple of $m$ and $n$, respectively. For real $y, z$, however, $(y, z)$ denotes the open interval on the real line with endpoints $y$ and $z$. The meaning is always clear from the context. Instead of $m \equiv n(\bmod k)$ we shall write for simplicity $m \equiv n(k)$. We shall also use the notation $e(t)=$ $\exp (2 \pi i t)$. The letter $c$ denotes some positive real number, not the same in all appearances. This convention allows us to write

$$
(\log t) e^{-c \sqrt{\log t}} \ll e^{-c \sqrt{\log t}},
$$

for example.

We define

$$
\begin{aligned}
& H=\frac{\sqrt{x}}{(\log x)^{16 A+100}}, \quad Q=(\log x)^{4 A+20}, \quad \tau=x Q^{-1}, \\
& E_{1}=\bigcup_{q \leq Q} \bigcup_{\substack{a=0 \\
(a, q)=1}}^{q-1}\left(\frac{a}{q}-\frac{1}{q \tau}, \frac{a}{q}+\frac{1}{q \tau}\right), \quad E_{2}=\left(-\frac{1}{\tau}, 1-\frac{1}{\tau}\right) \backslash E_{1}, \\
& S_{k}(\alpha)=\sum_{\substack{x<p \leq 3 x \\
p \equiv-2(k)}} e(\alpha p), \quad S(\alpha)=S_{1}(\alpha), \quad V(\alpha)=\sum_{x<m \leq 3 x} \frac{e(\alpha m)}{\log m}, \\
& \mathcal{E}=\sum_{\substack{k_{1}, k_{2} \leq H \\
\left(k_{1} k_{2}, 2\right)=1}} \sum_{D_{k_{1}, k_{2}}(x)-\frac{\sigma_{0} \gamma(x)}{\varphi\left(k_{1}\right) \varphi\left(k_{2}\right)}} \prod_{p \mid\left(k_{1}, k_{2}\right)} \frac{p-1}{p-2} \mid .
\end{aligned}
$$

3. Proof of the corollaries. Suppose that $\mu^{2}(k)=1$ and $M_{1}, M_{2}$ are integers. The following identity holds:

$$
\mu(k) \sum_{\substack{\left[k_{1}, k_{2}\right]=k \\ k_{1}\left|M_{1} \\ k_{2}\right| M_{2}}} \mu\left(k_{1}\right) \mu\left(k_{2}\right)= \begin{cases}1 & \text { if } k \mid M_{1} M_{2}, \\ 0 & \text { if } k \nmid M_{1} M_{2} .\end{cases}
$$

A similar identity has been stated in [1, Lemma 8]. For convenience we present a short proof. 
If $k \nmid M_{1} M_{2}$ the equality (4) is obvious. Suppose that $k \mid M_{1} M_{2}$. We have

$$
\begin{gathered}
\mu(k) \sum_{\substack{\left[k_{1}, k_{2}\right]=k \\
k_{1}\left|M_{1} \\
k_{2}\right| M_{2}}} \mu\left(k_{1}\right) \mu\left(k_{2}\right)=\mu(k) \sum_{\substack{k_{1}\left|k \\
k_{1}\right| M_{1}}} \sum_{\substack{d\left|k_{1} \\
k d / k_{1}\right| M_{2}}} \mu\left(k_{1}\right) \mu\left(\frac{k d}{k_{1}}\right) \\
=\sum_{\substack{k_{1} \mid\left(M_{1}, k\right) \\
M_{2} k_{1} \equiv 0(k)}} \sum_{d \mid\left(k_{1}, M_{2} k_{1} / k\right)} \mu(d)=\sum_{\substack{k_{1} \mid\left(M_{1}, k\right) \\
M_{2} k_{1} \equiv 0(k) \\
\left(k_{1}, M_{2} k_{1} / k\right)=1}} 1=1,
\end{gathered}
$$

since the only integer which satisfies the conditions imposed in the last sum is $k_{1}=k /\left(k, M_{2}\right)$. This completes the proof of (4).

Using (4) we get

$$
\begin{aligned}
J_{k}(x) & =\sum_{\substack{x<p_{1}, p_{2}, p_{3} \leq 3 x \\
p_{1}+p_{2}=2 p_{3}}} \mu(k) \sum_{\substack{\left[k_{1}, k_{2}\right]=k \\
k_{1}\left|\left(p_{1}+2\right) \\
k_{2}\right|\left(p_{2}+2\right)}} \mu\left(k_{1}\right) \mu\left(k_{2}\right) \\
& =\mu(k) \sum_{\left[k_{1}, k_{2}\right]=k} \mu\left(k_{1}\right) \mu\left(k_{2}\right) D_{k_{1}, k_{2}}(x) .
\end{aligned}
$$

Suppose that $\mu^{2}(k)=\mu^{2}(l)=1$ and $(k, 2)=(l, 2)=1$. We define

$$
t(l)=\prod_{p \mid l} \frac{p-1}{p-2}, \quad \varrho(l)=\frac{\mu(l) t(l)}{\varphi(l)}
$$

and

$$
L(k)=\mu(k) \sum_{\left[k_{1}, k_{2}\right]=k} \frac{\mu\left(k_{1}\right) \mu\left(k_{2}\right)}{\varphi\left(k_{1}\right) \varphi\left(k_{2}\right)} t\left(\left(k_{1}, k_{2}\right)\right) .
$$

It is clear that

$$
\begin{aligned}
L(k) & =\frac{\mu(k)}{t(k)} \sum_{\left[k_{1}, k_{2}\right]=k} \frac{\mu\left(k_{1}\right) \mu\left(k_{2}\right) t\left(k_{1}\right) t\left(k_{2}\right)}{\varphi\left(k_{1}\right) \varphi\left(k_{2}\right)}=\frac{\mu(k)}{t(k)} \sum_{\left[k_{1}, k_{2}\right]=k} \varrho\left(k_{1}\right) \varrho\left(k_{2}\right) \\
& =\frac{\mu(k)}{t(k)} \sum_{k_{1} \mid k} \sum_{d \mid k_{1}} \varrho\left(k_{1}\right) \varrho\left(\frac{k d}{k_{1}}\right)=\frac{\mu(k) \varrho(k)}{t(k)} \sum_{k_{1} \mid k} \sum_{d \mid k_{1}} \varrho(d) \\
& =\frac{\mu(k) \varrho(k)}{t(k)} \sum_{d \mid k} \varrho(d) \tau\left(\frac{k}{d}\right)=\frac{\mu(k) \varrho(k) \tau(k)}{t(k)} \sum_{d \mid k} \frac{\varrho(d)}{\tau(d)} \\
& =\frac{\mu(k) \varrho(k) \tau(k)}{t(k)} \prod_{p \mid k}\left(1+\frac{\varrho(p)}{2}\right) .
\end{aligned}
$$


Using the definitions of $\varrho(l)$ and $t(l)$ we easily compute

$$
L(k)=\prod_{p \mid k} \frac{2 p-5}{(p-1)(p-2)} .
$$

Now we apply (3), (5)-(7) and the Theorem to obtain

$$
\begin{aligned}
& \sum_{\substack{k \leq H \\
(k, 2)=1}} \mu^{2}(k)\left|J_{k}(x)-\sigma_{0} \gamma(x) \prod_{p \mid k} \frac{2 p-5}{(p-1)(p-2)}\right| \\
& =\sum_{\substack{k \leq H \\
(k, 2)=1}} \mu^{2}(k) \\
& \quad \times\left|\mu(k) \sum_{\left[k_{1}, k_{2}\right]=k} \mu\left(k_{1}\right) \mu\left(k_{2}\right)\left(D_{k_{1}, k_{2}}(x)-\frac{\sigma_{0} \gamma(x)}{\varphi\left(k_{1}\right) \varphi\left(k_{2}\right)} \prod_{p \mid\left(k_{1}, k_{2}\right)} \frac{p-1}{p-2}\right)\right| \\
& \leq \sum_{\substack{k_{1}, k_{2} \leq H \\
\left(k_{1} k_{2}, 2\right)=1}}\left|D_{k_{1}, k_{2}}(x)-\frac{\sigma_{0} \gamma(x)}{\varphi\left(k_{1}\right) \varphi\left(k_{2}\right)} \prod_{p \mid\left(k_{1}, k_{2}\right)} \frac{p-1}{p-2}\right| \ll \frac{x^{2}}{(\log x)^{A}} .
\end{aligned}
$$

Corollary 1 is proved.

Consider the sequence

$$
\mathcal{A}=\left\{\left(p_{1}+2\right)\left(p_{2}+2\right) \mid x<p_{1}, p_{2} \leq 3 x,\left(p_{1}+p_{2}\right) / 2 \text { prime }\right\}
$$

and let $\mathcal{B}$ be the set of odd primes. Define

$$
X=\sigma_{0} \gamma(x), \quad \omega(k)=k \prod_{p \mid k} \frac{2 p-5}{(p-1)(p-2)} .
$$

We apply Theorem 10.3 of [3] choosing $\kappa=2, \alpha=1 / 4, \mu=4.1, \zeta=0.4$. It is clear that we may get rid the extra factor $3^{\nu(d)}$ in the condition $R(\kappa, \alpha)$ using, for example, the Cauchy inequality. We obtain

$$
\left|\left\{\mathrm{P}_{9}: \mathrm{P}_{9} \in \mathcal{A}\right\}\right| \gg \frac{x^{2}}{\log ^{5} x} .
$$

Since the contribution of the terms for which $p_{1}=p_{2}$ is at most $\mathcal{O}(x)$, the last estimate proves Corollary 2.

4. Proof of the Theorem. It is clear that

$$
D_{k_{1}, k_{2}}(x)=\int_{-1 / \tau}^{1-1 / \tau} S_{k_{1}}(\alpha) S_{k_{2}}(\alpha) S(-2 \alpha) d \alpha=D_{k_{1}, k_{2}}^{(1)}(x)+D_{k_{1}, k_{2}}^{(2)}(x),
$$


where

$$
D_{k_{1}, k_{2}}^{(i)}(x)=\int_{E_{i}} S_{k_{1}}(\alpha) S_{k_{2}}(\alpha) S(-2 \alpha) d \alpha, \quad i=1,2 .
$$

Consequently,

$$
\mathcal{E} \leq \mathcal{E}_{1}+\mathcal{E}_{2}
$$

where

$$
\begin{aligned}
\mathcal{E}_{1} & =\sum_{\substack{k_{1}, k_{2} \leq H \\
\left(k_{1} k_{2}, 2\right)=1}}\left|D_{k_{1}, k_{2}}^{(1)}(x)-\frac{\sigma_{0} \gamma(x)}{\varphi\left(k_{1}\right) \varphi\left(k_{2}\right)} \prod_{p \mid\left(k_{1}, k_{2}\right)} \frac{p-1}{p-2}\right|, \\
\mathcal{E}_{2} & =\sum_{\substack{k_{1}, k_{2} \leq H \\
\left(k_{1} k_{2}, 2\right)=1}} \sum_{\substack{k_{1}, k_{2} \\
(2)}}^{(2)}(x) \mid .
\end{aligned}
$$

The proof of the Theorem follows from (3), (8)-(10) and from the inequalities

$$
\mathcal{E}_{1} \ll \frac{x^{2}}{(\log x)^{A}}, \quad \mathcal{E}_{2} \ll \frac{x^{2}}{(\log x)^{A}} .
$$

4.1. The estimate of $\mathcal{E}_{1}$. We have

$$
D_{k_{1}, k_{2}}^{(1)}(x)=\sum_{q \leq Q} \sum_{\substack{a=0 \\(a, q)=1}}^{q-1} I(a, q)
$$

where

$$
I(a, q)=\int_{-1 /(q \tau)}^{1 /(q \tau)} S_{k_{1}}\left(\frac{a}{q}+\alpha\right) S_{k_{2}}\left(\frac{a}{q}+\alpha\right) S\left(-2\left(\frac{a}{q}+\alpha\right)\right) d \alpha .
$$

If

$$
q \leq Q, \quad(a, q)=1, \quad|\alpha| \leq \frac{1}{q \tau}
$$

then we have

$$
S\left(-2\left(\frac{a}{q}+\alpha\right)\right)=\frac{h(q)}{\varphi(q)} V(-2 \alpha)+\mathcal{O}\left(x e^{-c \sqrt{\log x}}\right),
$$

where

$$
h(q)=\sum_{\substack{m=1 \\(m, q)=1}}^{q} e\left(\frac{2 m}{q}\right)=\frac{\mu\left(\frac{q}{(q, 2)}\right)}{\varphi\left(\frac{q}{(q, 2)}\right)} \cdot \varphi(q)
$$

(the proof is similar to that of $[6$, Lemma $3, \mathrm{X}]$ ).

Consider $S_{k}(a / q+\alpha)$ for $a, q, \alpha$ satisfying (13). We are not able to find an asymptotic formula for that sum for a particular large $k$ (unless we use 
some hypotheses which have not been proved yet). We shall find, however, an asymptotic formula with an error term which is small on average.

We have

$$
S_{k}\left(\frac{a}{q}+\alpha\right)=\sum_{\substack{1 \leq m \leq q \\(m, q)=1 \\ m \equiv-2((k, q))}} e\left(\frac{a m}{q}\right) T(\alpha)
$$

where

$$
T(\alpha)=\sum_{\substack{x<p \leq 3 x \\ p \equiv-2(k) \\ p \equiv m(q)}} e(\alpha p) .
$$

Using the elementary theory of congruences one may easily prove that if the integers $k, m, q$ satisfy $(k, 2)=(m, q)=1$ and $m \equiv-2((k, q))$ then there exists an integer $f=f(k, m, q)$ such that $(f,[k, q])=1$ and such that for any integer $n$ the congruence $n \equiv f([k, q])$ is equivalent to the system $n \equiv-2(k), n \equiv m(q)$. Hence we have

$$
T(\alpha)=\sum_{\substack{x<p \leq 3 x \\ p \equiv f([\overline{[k}, q])}} e(\alpha p) .
$$

We define

$$
\Delta(t, h)=\max _{y \leq t} \max _{(l, h)=1}\left|\sum_{\substack{p \leq y \\ p \equiv l(h)}} \log p-\frac{y}{\varphi(h)}\right| .
$$

Using Abel's formula we obtain

$$
\begin{aligned}
T(\alpha)= & -\int_{x}^{3 x}\left(\sum_{\substack{x<p \leq t \\
p \equiv f([k, q])}} \log p\right) \frac{d}{d t}\left(\frac{e(\alpha t)}{\log t}\right) d t+\left(\sum_{\substack{x<p \leq 3 x \\
p \equiv f([k, q])}} \log p\right) \frac{e(3 \alpha x)}{\log 3 x} \\
= & -\int_{x}^{3 x}\left(\frac{t-x}{\varphi([k, q])}+\mathcal{O}(\Delta(3 x,[k, q]))\right) \frac{d}{d t}\left(\frac{e(\alpha t)}{\log t}\right) d t \\
& +\left(\frac{2 x}{\varphi([k, q])}+\mathcal{O}(\Delta(3 x,[k, q]))\right) \frac{e(3 \alpha x)}{\log 3 x} \\
= & \frac{1}{\varphi([k, q])}\left(-\int_{x}^{3 x}(t-x) \frac{d}{d t}\left(\frac{e(\alpha t)}{\log t}\right) d t+2 x \frac{e(3 \alpha x)}{\log 3 x}\right) \\
& +\mathcal{O}((1+|\alpha| x) \Delta(3 x,[k, q]))
\end{aligned}
$$

We integrate by parts, then use (13) and the well-known formula 


$$
\int_{x}^{3 x} \frac{e(\alpha t)}{\log t} d t=V(\alpha)+\mathcal{O}(1)
$$

to get

$$
T(\alpha)=\frac{V(\alpha)}{\varphi([k, q])}+\mathcal{O}\left(\frac{Q}{q} \Delta(3 x,[k, q])\right) .
$$

We substitute this expression for $T(\alpha)$ in (15) and we find that under the condition (13) we have

$$
S_{k}\left(\frac{a}{q}+\alpha\right)=\frac{c_{k}(a, q)}{\varphi([k, q])} V(\alpha)+\mathcal{O}(Q \Delta(3 x,[k, q])),
$$

where

$$
c_{k}(a, q)=\sum_{\substack{1 \leq m \leq q \\(m, q)=1 \\ m \equiv-2((k, q))}} e\left(\frac{a m}{q}\right) .
$$

An explicit formula for the quantity $c_{k}(a, q)$ is found in [7, p. 218]. It implies that

$$
\left|c_{k}(a, q)\right| \leq 1 .
$$

Furthermore, we shall use the trivial estimates

$$
\left|S_{k}\left(\frac{a}{q}+\alpha\right)\right| \ll \frac{x}{k}, \quad|V(\alpha)| \ll \frac{x}{\log x}, \quad|h(q)| \ll 1 .
$$

From (14), (16), (18), (19) and the well-known estimate $\varphi(n) \gg n(\log \log n)^{-1}$ we get

$$
\begin{aligned}
S_{k_{1}}\left(\frac{a}{q}+\alpha\right) S_{k_{2}}\left(\frac{a}{q}+\alpha\right) S\left(-2\left(\frac{a}{q}+\alpha\right)\right) \\
=S_{k_{1}}\left(\frac{a}{q}+\alpha\right) S_{k_{2}}\left(\frac{a}{q}+\alpha\right) \frac{h(q)}{\varphi(q)} V(-2 \alpha)+\mathcal{O}\left(\frac{x^{3}}{k_{1} k_{2}} e^{-c \sqrt{\log x}}\right) \\
=S_{k_{1}}\left(\frac{a}{q}+\alpha\right) \frac{h(q)}{\varphi(q)} \cdot \frac{c_{k_{2}}(a, q)}{\varphi\left(\left[k_{2}, q\right]\right)} V(\alpha) V(-2 \alpha) \\
\quad+\mathcal{O}\left(\frac{Q x^{2}}{q k_{1}} \Delta\left(3 x,\left[k_{2}, q\right]\right)\right)+\mathcal{O}\left(\frac{x^{3}}{k_{1} k_{2}} e^{-c \sqrt{\log x}}\right) \\
=\frac{h(q) c_{k_{1}}(a, q) c_{k_{2}}(a, q)}{\varphi(q) \varphi\left(\left[k_{1}, q\right]\right) \varphi\left(\left[k_{2}, q\right]\right)} V^{2}(\alpha) V(-2 \alpha)+\mathcal{O}\left(\frac{x^{3}}{k_{1} k_{2}} e^{-c \sqrt{\log x}}\right) \\
\quad+\mathcal{O}\left(\frac{Q x^{2}}{q k_{2}} \Delta\left(3 x,\left[k_{1}, q\right]\right)\right)+\mathcal{O}\left(\frac{Q x^{2}}{q k_{1}} \Delta\left(3 x,\left[k_{2}, q\right]\right)\right) .
\end{aligned}
$$


For the integral $I(a, q)$ defined by (12), we find

$$
\begin{aligned}
I(a, q)= & \frac{h(q) c_{k_{1}}(a, q) c_{k_{2}}(a, q)}{\varphi(q) \varphi\left(\left[k_{1}, q\right]\right) \varphi\left(\left[k_{2}, q\right]\right)} \int_{-1 /(q \tau)}^{1 /(q \tau)} V^{2}(\alpha) V(-2 \alpha) d \alpha \\
& +\mathcal{O}\left(\frac{x Q^{2}}{k_{2} q^{2}} \Delta\left(3 x,\left[k_{1}, q\right]\right)\right)+\mathcal{O}\left(\frac{x Q^{2}}{k_{1} q^{2}} \Delta\left(3 x,\left[k_{2}, q\right]\right)\right) \\
& +\mathcal{O}\left(\frac{x^{2}}{k_{1} k_{2}} e^{-c \sqrt{\log x}}\right) .
\end{aligned}
$$

We also have

$$
\int_{-1 /(q \tau)}^{1 /(q \tau)} V^{2}(\alpha) V(-2 \alpha) d \alpha=\gamma(x)+\mathcal{O}\left(q^{2} \tau^{2}\right)
$$

(the proof is analogous to that in $[6$, Lemma 4, X]). Using (18)-(21) we find that

$$
\begin{aligned}
I(a, q)= & \frac{h(q) c_{k_{1}}(a, q) c_{k_{2}}(a, q)}{\varphi(q) \varphi\left(\left[k_{1}, q\right]\right) \varphi\left(\left[k_{2}, q\right]\right)} \gamma(x)+\mathcal{O}\left(\frac{q^{2} \tau^{2}}{\varphi(q) \varphi\left(\left[k_{1}, q\right]\right) \varphi\left(\left[k_{2}, q\right]\right)}\right) \\
& +\mathcal{O}\left(\frac{x Q^{2}}{k_{2} q^{2}} \Delta\left(3 x,\left[k_{1}, q\right]\right)\right)+\mathcal{O}\left(\frac{x Q^{2}}{k_{1} q^{2}} \Delta\left(3 x,\left[k_{2}, q\right]\right)\right) \\
& +\mathcal{O}\left(\frac{x^{2}}{k_{1} k_{2}} e^{-c \sqrt{\log x}}\right) .
\end{aligned}
$$

Set

$$
\begin{aligned}
b_{k_{1}, k_{2}}(q) & =\sum_{\substack{a=0 \\
(a, q)=1}}^{q-1} c_{k_{1}}(a, q) c_{k_{2}}(a, q), \\
\lambda_{k_{1}, k_{2}}(q) & =\frac{h(q) b_{k_{1}, k_{2}}(q) \varphi\left(\left(k_{1}, q\right)\right) \varphi\left(\left(k_{2}, q\right)\right)}{\varphi^{3}(q)} .
\end{aligned}
$$

From (11), (22)-(24) and the well-known formula

$$
\varphi([k, q]) \varphi((k, q))=\varphi(k) \varphi(q)
$$

we get

$$
\begin{aligned}
D_{k_{1}, k_{2}}^{(1)}(x)= & \frac{\gamma(x)}{\varphi\left(k_{1}\right) \varphi\left(k_{2}\right)} \sum_{q \leq Q} \lambda_{k_{1}, k_{2}}(q)+\mathcal{O}\left(\tau^{2}(\log x) \sum_{q \leq Q} \frac{q^{2}}{\left[k_{1}, q\right]\left[k_{2}, q\right]}\right) \\
& +\mathcal{O}\left(x Q^{2} \sum_{q \leq Q} \frac{\Delta\left(3 x,\left[k_{1}, q\right]\right)}{k_{2} q}\right) \\
& +\mathcal{O}\left(x Q^{2} \sum_{q \leq Q} \frac{\Delta\left(3 x,\left[k_{2}, q\right]\right)}{k_{1} q}\right)+\mathcal{O}\left(\frac{x^{2}}{k_{1} k_{2}} e^{-c \sqrt{\log x}}\right) .
\end{aligned}
$$


Consider the function $b_{k_{1}, k_{2}}(q)$. From (18) and (23) we have

$$
\left|b_{k_{1}, k_{2}}(q)\right| \leq \varphi(q) \text {. }
$$

It is not difficult to see that $b_{k_{1}, k_{2}}(q)$ is multiplicative with respect to $q$ and that for prime $p$ we have

$$
b_{k_{1}, k_{2}}(p)= \begin{cases}p-1 & \text { if } p \nmid k_{1}, p \nmid k_{2}, \\ 1 & \text { if } p \mid k_{1}, p \nmid k_{2}, \\ 1 & \text { if } p \nmid k_{1}, p \mid k_{2}, \\ -1 & \text { if } p\left|k_{1}, p\right| k_{2} .\end{cases}
$$

We also have $b_{k_{1}, k_{2}}(4)=0$. Therefore the function $\lambda_{k_{1}, k_{2}}(q)$ defined by $(24)$ is multiplicative with respect to $q$ and $\lambda_{k_{1}, k_{2}}\left(p^{l}\right)=0$ if $l \geq 2$. We apply Euler's identity (see [4, Theorem 286]) and also (19), (26), (27) and the definition of $\sigma_{0}$. After some calculations we get

$$
\sum_{q \leq Q} \lambda_{k_{1}, k_{2}}(q)=\sigma_{0} \prod_{p \mid\left(k_{1}, k_{2}\right)} \frac{p-1}{p-2}+\mathcal{O}\left(\sum_{q>Q} \frac{\left(k_{1}, q\right)\left(k_{2}, q\right)}{\varphi^{2}(q)}\right) .
$$

From (25), (28) and the trivial estimate

$$
\gamma(x) \ll \frac{x^{2}}{\log ^{3} x}
$$

we obtain

$$
\begin{aligned}
D_{k_{1}, k_{2}}^{(1)}(x)= & \frac{\sigma_{0} \gamma(x)}{\varphi\left(k_{1}\right) \varphi\left(k_{2}\right)} \prod_{p \mid\left(k_{1}, k_{2}\right)} \frac{p-1}{p-2}+\mathcal{O}\left(x^{2} \sum_{q>Q} \frac{\left(k_{1}, q\right)\left(k_{2}, q\right) \log q}{k_{1} k_{2} q^{2}}\right) \\
& +\mathcal{O}\left(x Q^{2} \sum_{q \leq Q} \frac{\Delta\left(3 x,\left[k_{1}, q\right]\right)}{k_{2} q}\right)+\mathcal{O}\left(x Q^{2} \sum_{q \leq Q} \frac{\Delta\left(3 x,\left[k_{2}, q\right]\right)}{k_{1} q}\right) \\
& +\mathcal{O}\left(\tau^{2}(\log x) \sum_{q \leq Q} \frac{q^{2}}{\left[k_{1}, q\right]\left[k_{2}, q\right]}\right)+\mathcal{O}\left(\frac{x^{2}}{k_{1} k_{2}} e^{-c \sqrt{\log x}}\right) .
\end{aligned}
$$

Using (9) and (29) we find

$$
\mathcal{E}_{1} \ll x Q^{2} \Sigma_{1}+\tau^{2}(\log x) \Sigma_{2}+x^{2} \Sigma_{3}+x^{2} e^{-c \sqrt{\log x}},
$$

where

$$
\begin{aligned}
\Sigma_{1} & =\sum_{k_{1}, k_{2} \leq H} \sum_{q \leq Q} \frac{\Delta\left(3 x,\left[k_{2}, q\right]\right)}{k_{1} q}, \quad \Sigma_{2}=\sum_{k_{1}, k_{2} \leq H} \sum_{q \leq Q} \frac{q^{2}}{\left[k_{1}, q\right]\left[k_{2}, q\right]}, \\
\Sigma_{3} & =\sum_{k_{1}, k_{2} \leq H} \sum_{q>Q} \frac{\left(k_{1}, q\right)\left(k_{2}, q\right) \log q}{k_{1} k_{2} q^{2}} .
\end{aligned}
$$


Consider $\Sigma_{1}$. We have

$$
\Sigma_{1} \ll(\log x) \sum_{k \leq H} \sum_{q \leq Q} \frac{\Delta(3 x,[k, q])}{q}=(\log x) \sum_{h \leq H Q} \Delta(3 x, h) \eta(h),
$$

where

$$
\eta(h)=\sum_{\substack{k \leq H \\[k, q]=h}} \sum_{\substack{q \leq Q \\ q}} \frac{1}{q} \sum_{d \leq Q} \sum_{\substack{k \leq H \\[k, q]=h \\(k, q)=d}} \sum_{q \leq Q} \frac{1}{q} \leq \sum_{d \leq Q} \sum_{\substack{q \leq Q \\ q \equiv 0(d)}} \frac{1}{q} \ll \log ^{2} x .
$$

Hence

$$
\Sigma_{1} \ll\left(\log ^{3} x\right) \sum_{h \leq H Q} \Delta(3 x, h) .
$$

Now we use the definitions of $H, Q$ and the Bombieri-Vinogradov theorem (see [2, Chapter 28], for example) and we find

$$
\Sigma_{1} \ll \frac{x}{(\log x)^{12 A+72}} .
$$

We now treat $\Sigma_{2}$. We have

$$
\begin{aligned}
\Sigma_{2} & =\sum_{d_{1}, d_{2} \leq Q} d_{1} d_{2} \sum_{\begin{array}{c}
k_{1}, k_{2} \leq H \\
\left(k_{1}, q\right)=d_{1} \\
\left(k_{2}, q\right)=d_{2}
\end{array}} \sum_{q \leq Q} \frac{1}{k_{1} k_{2}} \\
& \leq Q \sum_{d_{1}, d_{2} \leq Q} \frac{d_{1} d_{2}}{\left[d_{1}, d_{2}\right]} \sum_{\begin{array}{c}
k_{1}, k_{2} \leq H \\
k_{1} \equiv 0\left(d_{1}\right) \\
k_{2} \equiv 0\left(d_{2}\right)
\end{array}} \frac{1}{k_{1} k_{2}} \ll Q\left(\log ^{2} x\right) \Sigma^{*},
\end{aligned}
$$

where

$$
\begin{aligned}
\Sigma^{*} & =\sum_{d_{1}, d_{2} \leq Q} \frac{1}{\left[d_{1}, d_{2}\right]}=\sum_{d \leq Q} \sum_{\substack{d_{1}, d_{2} \leq Q \\
\left(d_{1}, d_{2}\right)=d}} \frac{d}{d_{1} d_{2}} \\
& \leq \sum_{d \leq Q} \frac{1}{d} \sum_{d_{1} \leq Q / d} \frac{1}{d_{1}} \sum_{d_{2} \leq Q / d} \frac{1}{d_{2}} \ll \log ^{3} x .
\end{aligned}
$$

Hence

$$
\Sigma_{2} \ll Q \log ^{5} x
$$

To complete the estimate of $\mathcal{E}_{1}$ we have to consider $\Sigma_{3}$. Obviously

$$
\Sigma_{3}=\sum_{d_{1}, d_{2} \leq H} d_{1} d_{2} \sum_{\substack{k_{1}, k_{2} \leq H \\\left(q, k_{1}\right)=d_{1} \\\left(q, k_{2}\right)=d_{2}}} \sum_{\substack{q>Q \\ k_{1}}} \frac{\log q}{k_{1} k_{2} q^{2}}=\Sigma_{4}+\Sigma_{5},
$$


where

$$
\begin{aligned}
& \Sigma_{4}=\sum_{\begin{array}{c}
d_{1}, d_{2} \leq H \\
{\left[d_{1}, d_{2}\right]>Q}
\end{array}} d_{1} d_{2} \sum_{\substack{k_{1}, k_{2} \leq H \\
\left(q, k_{1}\right)=d_{1} \\
\left(q, k_{2}\right)=d_{2}}} \sum_{q>Q} \frac{\log q}{k_{1} k_{2} q^{2}} \\
& \Sigma_{5}=\sum_{\left[d_{1}, d_{2}\right] \leq Q} d_{1} d_{2} \sum_{\substack{k_{1}, k_{2} \leq H \\
\left(q, k_{1}\right)=d_{1} \\
\left(q, k_{2}\right)=d_{2}}} \sum_{q>Q} \frac{\log q}{k_{1} k_{2} q^{2}} .
\end{aligned}
$$

We have

$$
\begin{aligned}
\Sigma_{4} & \ll \sum_{\substack{d_{1}, d_{2} \leq H \\
\left[d_{1}, d_{2}\right]>Q}} d_{1} d_{2} \sum_{\substack{k_{1}, k_{2} \leq H \\
k_{1} \equiv 0\left(d_{1}\right) \\
k_{2} \equiv 0\left(d_{2}\right)}} \frac{1}{k_{1} k_{2}} \sum_{q>Q /\left[d_{1}, d_{2}\right]} \frac{\log \left(q\left[d_{1}, d_{2}\right]\right)}{q^{2}\left[d_{1}, d_{2}\right]^{2}} \\
& \ll(\log x) \sum_{\substack{d_{1}, d_{2} \leq H \\
\left[d_{1}, d_{2}\right]>Q}} \frac{1}{\left[d_{1}, d_{2}\right]^{2}} \sum_{k_{1} \leq H / d_{1}} \frac{1}{k_{1}} \sum_{k_{2} \leq H / d_{2}} \frac{1}{k_{2}} \sum_{q=1}^{\infty} \frac{(1+\log q)}{q^{2}} \\
& \ll\left(\log ^{3} x\right) \sum_{h>Q} \frac{1}{h^{2}} \sum_{\left[d_{1}, d_{2}\right]=h} 1 \ll\left(\log ^{3} x\right) \sum_{h>Q} \frac{\tau_{3}(h)}{h^{2}} \ll \frac{\log ^{4} x}{Q} .
\end{aligned}
$$

For the sum $\Sigma_{5}$ we find

$$
\Sigma_{5} \ll\left(\log ^{3} x\right) \sum_{\left[d_{1}, d_{2}\right] \leq Q} \frac{1}{\left[d_{1}, d_{2}\right]^{2}} \sum_{q>Q /\left[d_{1}, d_{2}\right]} \frac{\log q}{q^{2}} \ll \frac{\log ^{4} x}{Q} \cdot \Sigma^{*}
$$

where $\Sigma^{*}$ is defined by (32). Consequently,

$$
\Sigma_{5} \ll \frac{\log ^{7} x}{Q} .
$$

Finally, combining (30), (31), (33)-(36) and using the definitions of $Q$ and $\tau$ we get

$$
\mathcal{E}_{1} \ll \frac{x^{2}}{(\log x)^{A}} .
$$

4.2. The estimate of $\mathcal{E}_{2}$. It is clear that

$$
\mathcal{E}_{2} \leq \sum_{k_{1}, k_{2} \leq H}\left|\int_{E_{2}} S_{k_{1}}(\alpha) S_{k_{2}}(\alpha) S(-2 \alpha) d \alpha\right| .
$$


Using the definition of $S_{k_{2}}(\alpha)$ we get

$$
\begin{aligned}
\mathcal{E}_{2} & \leq \sum_{k_{1}, k_{2} \leq H} \sum_{\substack{x<p \leq 3 x \\
p \equiv-2\left(k_{2}\right)}}\left|\int_{E_{2}} S_{k_{1}}(\alpha) S(-2 \alpha) e(\alpha p) d \alpha\right| \\
& \leq \sum_{k_{1}, k_{2} \leq H} \sum_{\substack{(x+2) / k_{2}<r \leq(3 x+2) / k_{2} \\
\leq}}\left|\int_{E_{2}} S_{k_{1}}(\alpha) S(-2 \alpha) e(-2 \alpha) e\left(\alpha r k_{2}\right) d \alpha\right| \\
& \leq \sum_{k_{1} \leq H} \sum_{n \leq 3 x+2}\left(\sum_{k_{2} \leq H} 1\right) \\
& \left.\times \mid \int_{E_{2}} \sum_{\substack{(x+2) / k_{2}<r \leq(3 x+2) / k_{2} \\
r k_{2}=n}} 1\right) \\
& \leq \sum_{k \leq H} \sum_{n \leq 3 x+2} \tau(n)\left|\int_{E_{2}} S_{k}(\alpha) S(-2 \alpha) e(-2 \alpha) e(\alpha n) d \alpha\right|
\end{aligned}
$$

By Cauchy's inequality we get

$$
\mathcal{E}_{2} \leq\left(\sum_{k \leq H} \sum_{n \leq 3 x+2} \frac{\tau^{2}(n)}{k}\right)^{1 / 2}\left(\sum_{k \leq H} k \sum_{n \leq 3 x+2}\left|\int_{-1 / \tau}^{1-1 / \tau} f(\alpha) e(\alpha n) d \alpha\right|^{2}\right)^{1 / 2}
$$

where

$$
f(\alpha)= \begin{cases}S_{k}(\alpha) S(-2 \alpha) e(-2 \alpha) & \text { if } \alpha \in E_{2}, \\ 0 & \text { if } \alpha \in E_{1} .\end{cases}
$$

We now apply Bessel's inequality to obtain

$$
\begin{aligned}
\mathcal{E}_{2} & \ll x^{1 / 2}\left(\log ^{2} x\right)\left(\sum_{k \leq H} k \int_{E_{2}}\left|S_{k}(\alpha) S(2 \alpha)\right|^{2} d \alpha\right)^{1 / 2} \\
& \leq x^{1 / 2}\left(\log ^{2} x\right)\left(\left.\sum_{k \leq H} k \sum_{\substack{x<p_{1}, p_{2} \leq 3 x \\
p_{1} \equiv p_{2} \equiv-2(k)}}\left|\int_{E_{2}}\right| S(2 \alpha)\right|^{2} e\left(\left(p_{1}-p_{2}\right) \alpha\right) d \alpha \mid\right)^{1 / 2} \\
& =x^{1 / 2}\left(\log ^{2} x\right) \Sigma^{1 / 2}, \quad \text { say. }
\end{aligned}
$$

We have

$$
\begin{aligned}
\Sigma & =\left.\sum_{k \leq H} k \sum_{\substack{|r| \leq 2 x \\
r \equiv 0(k)}}\left(\sum_{\substack{x<p_{1}, p_{2} \leq 3 x \\
p_{1} \equiv p_{2} \equiv-2 \\
p_{1}-p_{2}=r}} 1\right)\left|\int_{E_{2}}\right| S(2 \alpha)\right|^{2} e(\alpha r) d \alpha \mid \\
& \left.\ll x \sum_{k \leq H} \sum_{\substack{|r| \leq 2 x \\
r \equiv 0(k)}}\left|\int_{E_{2}}\right| S(2 \alpha)\right|^{2} e(\alpha r) d \alpha \mid \\
& =x\left(\Sigma^{\prime}+\Sigma^{\prime \prime}\right),
\end{aligned}
$$


where

$$
\begin{aligned}
\Sigma^{\prime} & =\sum_{k \leq H}\left(\int_{E_{2}}|S(2 \alpha)|^{2} d \alpha\right), \\
\Sigma^{\prime \prime} & =\left.\sum_{k \leq H} \sum_{\substack{\leq \leq|r| \leq 2 x \\
r \equiv 0(k)}}\left|\int_{E_{2}}\right| S(2 \alpha)\right|^{2} e(\alpha r) d \alpha \mid .
\end{aligned}
$$

Obviously

$$
\Sigma^{\prime} \leq H \int_{0}^{1}|S(2 \alpha)|^{2} d \alpha \ll \frac{H x}{\log x} .
$$

By the Cauchy inequality we find

$$
\begin{aligned}
\Sigma^{\prime \prime} & \left.\ll \sum_{k \leq H} \sum_{\substack{1 \leq r \leq 2 x \\
r \equiv 0(k)}}\left|\int_{E_{2}}\right| S(2 \alpha)\right|^{2} e(r \alpha) d \alpha \mid \\
& =\left.\sum_{1 \leq r \leq 2 x}\left(\sum_{\substack{k \leq H \\
k \mid r}} 1\right)\left|\int_{E_{2}}\right| S(2 \alpha)\right|^{2} e(r \alpha) d \alpha \mid \\
& \left.\ll \sum_{1 \leq r \leq 2 x} \tau(r)\left|\int_{E_{2}}\right| S(2 \alpha)\right|^{2} e(r \alpha) d \alpha \mid \\
& \ll\left(\sum_{1 \leq r \leq 2 x} \tau^{2}(r)\right)^{1 / 2}\left(\sum_{1 \leq r \leq 2 x}\left|\int_{-1 / \tau}^{1-1 / \tau} g(\alpha) e(r \alpha) d \alpha\right|^{2}\right)^{1 / 2},
\end{aligned}
$$

where

$$
g(\alpha)= \begin{cases}|S(2 \alpha)|^{2} & \text { if } \alpha \in E_{2}, \\ 0 & \text { if } \alpha \in E_{1} .\end{cases}
$$

We again apply the Bessel inequality to obtain

$$
\begin{aligned}
\Sigma^{\prime \prime} & \ll x^{1 / 2}(\log x)^{3 / 2}\left(\int_{E_{2}}|S(2 \alpha)|^{4} d \alpha\right)^{1 / 2} \\
& \ll x^{1 / 2}(\log x)^{3 / 2} \sup _{\alpha \in E_{2}}|S(2 \alpha)|\left(\int_{0}^{1}|S(2 \alpha)|^{2} d \alpha\right)^{1 / 2} \\
& \ll x(\log x) \sup _{\alpha \in E_{2}}|S(2 \alpha)| .
\end{aligned}
$$

Using the definitions of $Q, \tau$ and $E_{2}$ we can prove in the same way as in $[6$, Theorem 3, X] that

$$
\sup _{\alpha \in E_{2}}|S(2 \alpha)| \ll \frac{x}{(\log x)^{2 A+7}} .
$$


From (37)-(41) we obtain

$$
\mathcal{E}_{2} \ll \frac{x^{2}}{(\log x)^{A}}
$$

The Theorem is proved.

Finally, the authors would like to thank the Ministry of Science and Education of Bulgaria for financial support under grant MM-430.

\section{References}

[1] J. Brüdern and E. Fouvry, Lagrange's Four Squares Theorem with almost prime variables, J. Reine Angew. Math. 454 (1994), 59-96.

[2] H. Daven port, Multiplicative Number Theory (revised by H. Montgomery), 2nd ed., Springer, 1980.

[3] H. Halberstam and H.-E. Richert, Sieve Methods, Academic Press, London, 1974.

[4] G. H. Hardy and E. M. Wright, An Introduction to the Theory of Numbers, 5th ed., Oxford Univ. Press, 1979.

[5] D. R. Heath-Brown, Three primes and an almost-prime in arithmetic progression, J. London Math. Soc. (2) 23 (1981), 396-414.

[6] A. A. Karatsuba, Principles of Analytic Number Theory, Nauka, Moscow, 1983 (in Russian).

[7] H. Maier and C. Pomerance, Unusually large gaps between consecutive primes, Trans. Amer. Math. Soc. 322 (1990), 201-237.

[8] D. I. Tolev, On the number of representations of an odd integer as a sum of three primes, one of which belongs to an arithmetic progression, Proc. Steklov Math. Inst., to appear.

[9] J. G. van der Corput, Über Summen von Primzahlen und Primzahlquadraten, Math. Ann. 116 (1939), 1-50.

[10] I. M. Vinogradov, Representation of an odd number as a sum of three primes, Dokl. Akad. Nauk SSSR 15 (1937), 169-172 (in Russian).

Department of Mathematics

Plovdiv University "P. Hilendarski"

"Tsar Asen" 24

Plovdiv 4000, Bulgaria

E-mail: tpeneva@ulcc.uni-plovdiv.bg dtolev@ulcc.uni-plovdiv.bg 